\title{
Características y pronóstico de los pacientes mayores con estancia muy prolongada en una Unidad de Cuidados Intensivos
}

\author{
L. SANTANA CABRERA, M. SÁNCHEZ-PALACIOS, E. HERNÁNDEZ MEDINA, \\ P. EUGENIO ROBAINA Y Á. VILLANUEVA-HERNÁNDEZ
}

Servicio de Medicina Intensiva. Hospital Universitario Insular de Gran Canaria. Las Palmas de Gran Canaria. España.

Objetivo. Describir las características y los factores pronósticos de los pacientes mayores ingresados de forma prolongada en nuestra Unidad de Cuidados Intensivos (UCl).

Diseño. Se trata de un análisis retrospectivo de datos recogidos prospectivamente durante 6 años consecutivos.

Ámbito. Una UCI polivalente del Hospital Universitario Insular de Gran Canaria.

Pacientes. El estudio se centra en pacientes adultos mayores de 70 años que permanecieron ingresados en la $\mathrm{UCl}$ durante un período igual o superior a 30 días.

Variables de interés principales. Se recogieron datos demográficos y diagnósticos de los pacientes al ingreso, Apache II y los días que precisaron de terapias de reemplazamiento renal (RRT) y de ventilación mecánica. También se estudió la mortalidad al año de los pacientes que sobrevivieron.

Resultados. Durante el período de estudio ingresaron 3.786 pacientes en la UCl. Ochocientos cincuenta y tres $(22,5 \%)$ tenían más de 70 años y sólo $42(4,92 \%)$ de ellos permanecieron en la UCI durante 30 o más días. Comparamos estos últimos con los pacientes mayores de 70 años pero con una estancia en la $\mathbf{U C l}$ inferior a $\mathbf{3 0}$ días y no encontramos diferencias estadísticamente significativas ni en la mortalidad en la $\mathrm{UCI}$, ni en el Apache II, la edad, el sexo o la necesidad de RRT.

Correspondencia: Dr. L. Santana Cabrera.

Servicio de Medicina Intensiva.

Hospital Universitario Insular de Gran Canaria

Avda. Marítima del Sur, s/n.

35016 Gran Canaria. Las Palmas de Gran Canaria. España.

Correo electrónico: lsancabx@gobiernodecanarias.org

Manuscrito aceptado el 3-VIII-2007.
El análisis multivariado mostró como variables independientes asociadas a la estancia prolongada solamente los días de ventilación mecánica ( $p<$ $0,05)$. Los pacientes de $\geq 70$ años y con una estancia $\geq \mathbf{3 0}$ días que sobrevivieron eran más mayores y $21(65,62 \%)$ estaban vivos al año.

Conclusiones. Los índices de mortalidad en los pacientes de $\geq 70$ años con una estancia $<0 \geq 30$ días en la $\mathrm{UCI}$ fueron similares. La supervivencia al año de los pacientes de $\geq 70$ años con una estancia $\geq 30$ días en la UCl fue alta. Los resultados del estudio justifican el cuidado de los pacientes ancianos con estancias prolongadas en $\mathrm{UCl}$.

PALABRAS CLAVE: anciano, Unidad de Cuidados Intensivos, estancia, pronóstico.

\section{CHARACTERISTICS AND PROGNOSIS OF PATIENTS WITH VERY LONG STAY IN AN INTENSIVE CARE UNIT}

Objective. To describe the characteristics and prognostic factors of elderly patients hospitalized for $\geq \mathbf{3 0}$ days in an Intensive Care Unit (ICU).

Design. Retrospective analysis of prospectively collected simple data over 6 consecutive years.

Setting. Polyvalent ICU of the Insular University Hospital in the Canary Islands (Spain).

Patients. Adult patients $\geq 70$ years who were hospitalized in the ICU for a period of $\mathbf{3 0}$ or more days.

Primary variables of interest. Demographic data, clinical diagnosis on ICU admission, Apache II, days of renal replacement therapy (RRT), days of mechanical ventilation and the outcome of the survivors one year later were collected. Mortality at one year of the surviving patients was studied.

Results. During the study period, 3,786 patients were admitted to the ICU. Of these, $853(22.5 \%)$ 
patients were $\geq 70$ years old and only $42(4.92 \%)$ of these patients remained in the ICU for $\geq \mathbf{3 0}$ days. We compared the latter with the $\geq 70$ year old patients whose stay in the ICU stay was < 30 days. No statistically significant differences in ICU mortality, Apache II, age, gender and the need for RRT were found. As independent variables associated with the long stay, the multivariate analysis showed only the days of mechanical ventilation ( $p$ $<0.05)$. The surviving patients $(\geq 70$ years old and whose stay in the ICU was $\geq 30$ days) were older and $21(65.62 \%)$ were still alive one year later.

Conclusions. ICU mortality rates in elderly patients with a stay $<$ or $\geq 30$ days in the ICU were comparable. Survival at one year of the $\geq 70$ yearold patients whose long-term intensive care unit stay was $\geq 30$ days was high. These results are sufficient in our unit to justify prolonged ICU care for elderly patients.

KEY WORDS: frail elderly, Intensive Care Units, length of stay, outcome.

\section{INTRODUCCIÓN}

La estancia prolongada en la Unidad de Cuidados Intensivos (UCI) se asocia con mayores tasas de mortalidad, lo cual conllevará un importante consumo de recursos asistenciales ${ }^{1}$. En los estudios publicados, la estancia prolongada no está definida de forma uniforme, así hay autores que la definen como una estancia mayor de 10 a 14 días $^{2,3}$ y otros se refieren a estancias mayores de 28 a 30 días $^{4-6}$.

La edad media de los pacientes que ingresan en la UCI se incrementa paralelamente con el aumento en la esperanza de vida de la población general ${ }^{7}$. Podríamos suponer que en cuanto a mortalidad, los pacientes más mayores ingresados en la UCI tendrían un peor pronóstico que los más jóvenes; sin embargo, el pronóstico de estos pacientes está más relacionado con la severidad de la enfermedad aguda ${ }^{8}$, mientras que la edad no es un predictor real del pronóstico de estos pacientes en la UCI, ya que incluso los mayores que sobreviven tienen un mejor pronóstico que los jóvenes $^{6}$. La edad en sí misma no debe ser un factor limitante en la aplicación del tratamiento intensivo a los pacientes ancianos, sino que hay que tener en cuenta la calidad de vida previa y posterior al ingreso en la UCI9.

No obstante, es frecuente que se planteen dudas sobre la idoneidad de seguir adelante cuando un paciente de edad avanzada se cronifica en la UCI, y por ello nuestro objetivo fue describir las características y el pronóstico de los pacientes mayores de 70 años que estuvieron hospitalizados durante más de 30 días en nuestra UCI.

\section{PACIENTES Y MÉTODO}

Se trata de un estudio retrospectivo observacional de los datos recogidos prospectivamente sobre los pacientes ingresados en nuestra UCI, de carácter polivalente y con una capacidad de 24 camas, perteneciente a un hospital terciario. Se incluyeron todos los pacientes ingresados durante el período comprendido entre enero de 2001 y diciembre de 2006. Se analizaron variables demográficas como la edad, el sexo, el tipo de paciente (coronario, médico, quirúrgico o traumatológico), Apache II al ingreso, los días de estancia en la UCI, la necesidad de técnicas como la ventilación mecánica o la depuración extrarrenal y los días que precisó de ellas durante su ingreso. También se estudió la mortalidad de estos pacientes en la UCI y en el hospital, así como al mes y al año del alta.

Se analizaron todos los pacientes ingresados durante $\geq 30$ días en la Unidad, y se compararon los vivos con los muertos y con los que tuvieron una estancia < 30 días; asimismo se estudiaron los pacientes de $\geq 70$ años según su estancia y su supervivencia mediante las pruebas de la «t» de Student o la de MannWhitney para comparar variables continuas y la prueba de Chi cuadrado o la de Fisher para comparar porcentajes, en los que se consideró significativa una $\mathrm{p}<0,05$.

Asimismo se estudió la influencia de diversos factores en la mortalidad mediante el análisis de regresión logística multivariada, expresando los resultados como odds ratio y el intervalo de confianza al 95\%, y se consideró una p significativa cuando era $\leq 0,05$.

\section{RESULTADOS}

Durante el período de estudio ingresaron en nuestra UCI 3.786 pacientes, de los cuales $853(22,5 \%)$ tenían $\geq 70$ años y, de ellos, $42(4,9 \%)$ permanecieron durante $\geq 30$ días.

En primer lugar estudiamos a todos los pacientes ingresados, independientemente de su edad, según fuese su estancia $<0 \geq 30$ días, y no encontramos diferencias estadísticamente significativas en la mortalidad entre ambos grupos, 19,4 frente a 22\%, a pesar de que el grupo que permaneció durante más tiempo en la UCI estaba más grave, según la puntuación Apache II al ingreso, y había precisado más días de ventilación mecánica y de terapias de reemplazamiento renal continuas (tabla 1). Cuando analizamos los pacientes con una estancia $\geq 30$ días según la mortalidad, no encontramos diferencias estadísticamente significativas en las variables estudiadas salvo en los días de ventilación mecánica, que fueron superiores en los fallecidos (48,2 frente a 32,7 días) (tabla 2).

Al comparar las diferencias de los pacientes de $\geq 70$ años según fuese su estancia $<$ o $\geq 30$ días, sólo encontramos diferencias estadísticamente significativas en la necesidad de ventilación mecánica $(\mathrm{p}=0,004)$ y en los días de depuración extrarrenal $(\mathrm{p}=0,03)$, mayores en el grupo de más estancia. La diferencia en la 
TABLA 1. Características de todos los pacientes ingresados según su estancia en la Unidad de Cuidados Intensivos

\begin{tabular}{|c|c|c|c|}
\hline & $<30$ días $(\mathrm{n}=3.650)$ & $\geq 30$ días $(\mathrm{n}=136)$ & $\mathrm{p}$ \\
\hline Edad, años (media \pm DE) & $55,39 \pm 10,13$ & $57,47 \pm 16,43$ & 0,14 \\
\hline Sexo femenino.$^{\circ}(\%)$ & $1.219(33,31 \%)$ & $41(30,14 \%)$ & 0,63 \\
\hline Apache II & $10,89 \pm-7,95$ & $15,53 \pm 6,29$ & $<0,001$ \\
\hline Estancia en la UCI, días & $4,38 \pm 5,75$ & $44,28 \pm 15,44$ & $<0,001$ \\
\hline \multicolumn{4}{|l|}{ Grupo diagnóstico al ingreso, n. ${ }^{\circ}(\%)$} \\
\hline Cardiovascular & $1.232(33,67 \%)$ & $12(8,95 \%)$ & $<0,001$ \\
\hline Médico & $1.061(28,99 \%)$ & $50(37,31 \%)$ & 0,19 \\
\hline Quirúrgico & $1.154(31,53 \%)$ & $60(44,77 \%)$ & 0,042 \\
\hline Trauma & $212(5,79 \%)$ & $12(8,96 \%)$ & 0,23 \\
\hline Ventilación mecánica durante la estancia en la UCI, n. ${ }^{\circ}(\%)$ & $1.654(45,31 \%)$ & $136(100 \%)$ & $<0,001$ \\
\hline Días de ventilación mecánica (media \pm DE) & $5,87 \pm 6,43$ & $36,28 \pm 17,62$ & $<0,001$ \\
\hline Terapias de reemplazamiento renal durante su estancia en la UCI, n. ${ }^{\circ}(\%)$ & $171(4,68 \%)$ & $11(8,08 \%)$ & \\
\hline Días con terapia de reemplazamiento renal (media $\pm \mathrm{DE})$ & $1,46 \pm 3,23$ & $13,18 \pm 12,80$ & 0,012 \\
\hline Mortalidad en la UCI, n. ${ }^{\circ}(\%)$ & $710(19,46 \%)$ & $30(22,05 \%)$ & 0,610 \\
\hline
\end{tabular}

DE: desviación estándar; UCI: Unidad de Cuidados Intensivos.

mortalidad entre ambos grupos no fue significativa, con una $\mathrm{p}=0,640$ (tabla 3$)$.

Cuando analizamos a los pacientes de $\geq 70$ años con una estancia prolongada en la UCI, según fallecieran o sobrevivieran, sólo encontramos diferencias significativas en la edad y en los días en que estuvieron sometidos a ventilación mecánica. En relación con la edad, el grupo de los supervivientes presentaba una edad superior: 75,1 frente a 73 años, $p=0,026$; y en relación con los días sometidos a ventilación mecánica, encontramos períodos más largos en los pacientes que fallecieron en la UCI $(53,4$ frente a 32 días, con una $\mathrm{p}=0,00142$ ), tal como podemos observar en la tabla 4.

También estudiamos la supervivencia al año de los pacientes de $\geq 70$ años que permanecieron $\geq 30$ días en la UCI, pero no encontramos en el momento de redactar el presente artículo ningún paciente vivo pendiente de seguimiento hasta un año. El resultado fue que $6(18,7 \%)$ fallecieron en el hospital tras el alta de la UCI, 5 en los primeros 15 días y 1 antes del mes; sin embargo, $21(65,6 \%)$ de los supervivientes estaban vivos al año (fig. 1). Dicho de otra forma, la supervivencia al año del total de este grupo de pacientes fue del $50 \%$ (21 vivos y 21 muertos).
El análisis multivariado mostró como variables independientes asociadas a la mortalidad de los pacientes de $\geq 70$ años con una estancia prolongada en la UCI, solamente los días de ventilación mecánica, con una $\mathrm{p}<0,05$.

\section{DISCUSIÓN}

Nuestro principal hallazgo es que más del $60 \%$ de los pacientes mayores de 70 años que permanecieron de forma prolongada, es decir más de 30 días, ingresados en la UCI y que sobrevivieron estaban vivos al año del alta. Además, podemos decir que la mortalidad en la UCI de estos pacientes es de aproximadamente el $23 \%$, y que no es estadísticamente significativa la diferencia con la mortalidad de los pacientes mayores que no permanecieron de forma prolongada en la UCI.

La mortalidad hospitalaria tras el alta de la UCI de los pacientes estudiados fue sólo del $18,7 \%$, cifra que es mucho menor en relación con otros estudios de pacientes con estancia prolongada en la $\mathrm{UCI}^{2,3}$.

Si comparamos nuestros resultados con los de un grupo español que estudió la supervivencia de pacientes mayores de 65 años que estuvieron ingresados

TABLA 2. Diferencias entre los pacientes con estancia $\geq \mathbf{3 0}$ días según la mortalidad

\begin{tabular}{|c|c|c|c|}
\hline & Vivos $(\mathrm{n}=30)$ & Muertos $(\mathrm{n}=106)$ & $\mathrm{p}$ \\
\hline Edad, años (media \pm DE) & $61,26 \pm 11,31$ & $56,39 \pm 17,50$ & 0,07 \\
\hline Sexo femenino.$^{\circ}(\%)$ & $11(36,66 \%)$ & $30(28,30 \%)$ & 0,67 \\
\hline Apache II & $15,76 \pm 5,91$ & $15,47 \pm 6,42$ & 0,82 \\
\hline Estancia en la UCI, días & $48,93 \pm 16,22$ & $42,97 \pm 15,03$ & 0,059 \\
\hline \multicolumn{4}{|l|}{ Grupo diagnóstico al ingreso, n. $^{\circ}(\%)$} \\
\hline Cardiovascular & $3(10 \%)$ & $9(8,65 \%)$ & 0,89 \\
\hline Médico & $11(36,66 \%)$ & $39(37,5 \%)$ & 0,84 \\
\hline Quirúrgico & $14(46,66 \%)$ & $46(44,23 \%)$ & 0,99 \\
\hline Trauma & $2(6,66 \%)$ & $10(9,6 \%)$ & 0,94 \\
\hline Ventilación mecánica durante su estancia en la UCI, n. ${ }^{\circ}(\%)$ & $30(100 \%)$ & $106(100 \%)$ & 0,98 \\
\hline Días de ventilación mecánica (media \pm DE) & $48,2 \pm 16,61$ & $32,78 \pm 16,40$ & $<0,0001$ \\
\hline Terapias de reemplazamiento renal durante su estancia en la UCI, n. ${ }^{\circ}(\%)$ & $5(16,6 \%)$ & $6(5,6 \%)$ & 0,16 \\
\hline Días (media \pm DE) & $21 \pm 12,64$ & $6,66 \pm 9,37$ & 0,058 \\
\hline
\end{tabular}

DE: desviación estándar; UCI: Unidad de Cuidados Intensivos. 
TABLA 3. Diferencias entre los pacientes de $\geq 70$ años ingresados según sea su estancia $<0 \geq 30$ días

\begin{tabular}{|c|c|c|c|}
\hline & $<30$ días $(\mathrm{n}=811)$ & $\geq 30$ días $(\mathrm{n}=42)$ & $\mathrm{p}$ \\
\hline Edad, años (media \pm DE) & $74,89 \pm 4,06$ & $74,60 \pm 3,94$ & 0,719 \\
\hline Sexo femenino.$^{\circ}(\%)$ & $254(33,82 \%)$ & $7(16 \%)$ & 0,160 \\
\hline Apache II & $14,86 \pm 7,87$ & $15,16 \pm 4,97$ & 0,709 \\
\hline Estancia en la UCI, días & $4,58 \pm 6,07$ & $46,59 \pm 14,23$ & $<0,001$ \\
\hline \multicolumn{4}{|l|}{ Grupo diagnóstico al ingreso, $\mathrm{n}^{\circ}(\%)$} \\
\hline Cardiovascular & $312(38,44 \%)$ & $5(11,90 \%)$ & 0,015 \\
\hline Médico & $248(30,53 \%)$ & $15(35,71 \%)$ & 0,730 \\
\hline Quirúrgico & $234(28,80 \%)$ & $18(42,85 \%)$ & 0,220 \\
\hline Trauma & $17(1,97 \%)$ & $4(9,52 \%)$ & 0,010 \\
\hline Ventilación mecánica durante la estancia en la UCI, n. ${ }^{\circ}(\%)$ & $405(49,93 \%)$ & $42(100 \%)$ & 0,004 \\
\hline Días de ventilación mecánica (media \pm DE) & $5,52 \pm 6,26$ & $37,21 \pm 18,28$ & $<0,001$ \\
\hline Terapias de reemplazamiento renal durante su estancia en la UCI, n. ${ }^{\circ}(\%)$ & $47(5,79 \%)$ & $3(7,14 \%)$ & 0,290 \\
\hline Días con terapia de reemplazamiento renal (media \pm DE) & $1,97 \pm 4,15$ & $7,67 \pm 6,65$ & 0,03 \\
\hline Mortalidad en la UCI, n. ${ }^{\circ}(\%)$ & $242(29,9 \%)$ & $10(23,8 \%)$ & 0,640 \\
\hline
\end{tabular}

DE: desviación estándar; UCI: Unidad de Cuidados Intensivos.

en Cuidados Intensivos ${ }^{10}$, éstos tuvieron una mortalidad hospitalaria tras el alta de la UCI del $10 \%$, que es significativamente menor que la de nuestros pacientes, aunque la estancia media en la UCI había sido mucho menor, 11 días. En este estudio concluyen que a pesar de que la mortalidad al año que encontraron fue alta $(51 \%)$, el $83 \%$ de los supervivientes era capaz de vivir de forma independiente, y que la mortalidad dependía del desarrollo de disfunción multiorgánica durante su estancia en la UCI y no de la edad.

El pronóstico de los pacientes con estancia prolongada en la UCI ha sido estudiado por algunos autores $^{11}$ que encontraron una mortalidad del $24,4 \%$, similar a la nuestra, que fue aproximadamente del $22 \%$; éstos encontraron una mayor relación con la mortalidad en los pacientes que tenían fallo multiorgánico.

Cuando comparamos nuestros pacientes mayores ( $\geq 70$ años) con estancia prolongada ( $\geq 30$ días) según fallecieran o sobrevivieran a la UCI, encontramos que los que fallecieron precisaron más días de ventilación mecánica, de forma que estos días de ventilación mecánica son una variable independiente asociada a la mortalidad $(\mathrm{p}<0,05)$. Sin embargo, cuando analizamos el total de pacientes, sin límite de edad, con una estancia $\geq 30$ días, los días de ventilación mecánica fueron significativamente mayores en los que sobrevivieron. La influencia de la ventilación mecánica prolongada en la mortalidad ha sido estudiada en algún trabajo ${ }^{12}$ que llega a la conclusión de que ésta se asociaba con una mayor mortalidad en la UCI y tras la UCI.

La estancia prolongada en la UCI está claro que va a consumir muchos más recursos ${ }^{1}$, pero esto no parece influir en la calidad de vida a largo plazo ${ }^{13-15}$. También la estancia prolongada tiene un gran impacto en la utilización de camas en la UCI, ya que la disponibilidad de éstas para los pacientes de corta estancia se verá reducida ${ }^{16}$.

Sería interesante encontrar una serie de indicadores precoces que nos pongan sobre aviso de que un paciente va a permanecer de forma prolongada en la UCI y, por tanto, va a generar un gran coste ${ }^{17,18}$. En este sentido, Higgins et $\mathrm{al}^{17}$ concluyen en su trabajo que parece que a la severidad de la enfermedad sólo se le implica parcialmente en la predicción de la estancia prolongada, que la edad es un factor menos relevante que la presencia de infección o la dependencia al ventilador a las 24 horas, y que la mayor estancia hospita-

TABLA 4. Características de los pacientes $\geq \mathbf{7 0}$ años ingresados durante $\mathbf{2} \mathbf{3 0}$ días en la Unidad de Cuidados Intensivos

\begin{tabular}{|c|c|c|c|}
\hline & $\operatorname{Vivos}(\mathrm{n}=32)$ & Muertos $(\mathrm{n}=10)$ & $\mathrm{p}$ \\
\hline Edad, años (media $\pm \mathrm{DE})$ & $75,18 \pm 4,29$ & $73 \pm 1,76$ & 0,026 \\
\hline Sexo femenino $n .^{\circ}(\%)$ & $7(21,87 \%)$ & $0(0 \%)$ & 0,83 \\
\hline Apache II & $15,03 \pm 4,93$ & $15,6 \pm 4,97$ & 0,75 \\
\hline Estancia en la UCI, días & $44,28 \pm 14,62$ & $54 \pm 10,27$ & 0,058 \\
\hline \multicolumn{4}{|l|}{ Grupo diagnóstico al ingreso, . $^{\circ}(\%)$} \\
\hline Cardiovascular & $5(15,62 \%)$ & $0(0 \%)$ & \\
\hline Médico & $13(40,62 \%)$ & $2(20 \%)$ & 0,62 \\
\hline Quirúrgico & $11(34,37 \%)$ & $7(70 \%)$ & 0,38 \\
\hline Trauma & $3(9,3 \%)$ & $1(10 \%)$ & 0,57 \\
\hline Ventilación mecánica durante la estancia en la UCI, n. ${ }^{\circ}(\%)$ & $32(100 \%)$ & $10(100 \%)$ & 0,79 \\
\hline Días de ventilación mecánica (media $\pm \mathrm{DE}$ ) & $32 \pm 17,34$ & $53,4 \pm 9,91$ & 0,00142 \\
\hline Terapias de reemplazamiento renal durante su estancia en la UCI, ${ }^{\circ}{ }^{\circ}(\%)$ & $1(3,12 \%)$ & $2(20 \%)$ & 0,34 \\
\hline
\end{tabular}

DE: desviación estándar; UCI: Unidad de Cuidados Intensivos. 


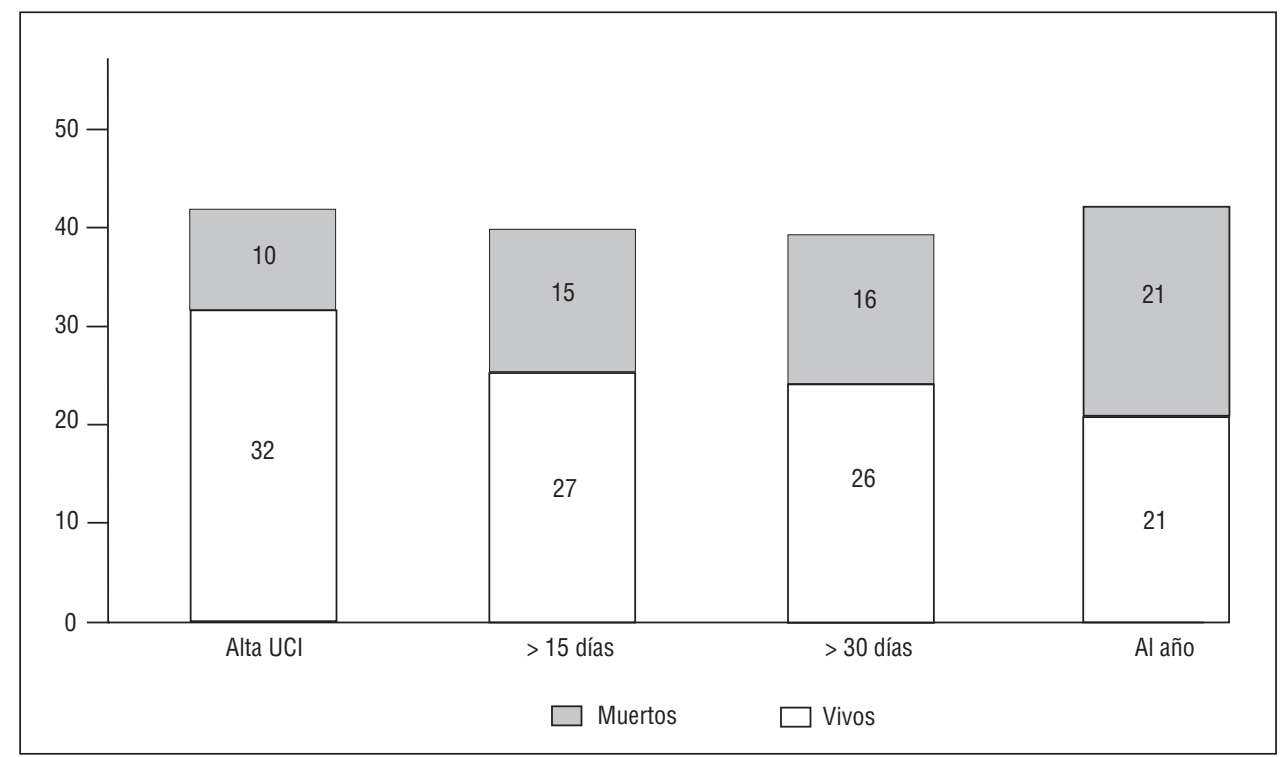

Figura 1. Supervivencia tras el alta de los pacientes $\geq 70$ años ingresados durante $\geq 30$ días en la Unidad de Cuidados Intensivos (UCI).

laria antes del ingreso en la UCI y la falta de un médico en la misma con dedicación exclusiva incrementarán la probabilidad de que un paciente permanezca más tiempo en esta Unidad. De todas formas, el esfuerzo para implementar sistemas de análisis de los costes en general, y de los de la calidad en particular, redundará en el beneficio de los actores del sistema sanitario, los ciudadanos, los profesionales, los gestores y los financiadores ${ }^{19}$.

Los pacientes mayores comportan, según algunas series, el $42-52 \%$ de los ingresos en la UCI y casi el $60 \%$ de ocupación de los días de la UCI; sin embargo, en nuestro estudio sólo supuso el $22,5 \%$ de los ingresos. Únicamente la edad no debería usarse como triage de los pacientes que deben ingresar en la UCI, sino que la decisión de ingresarlos debería basarse en sus co-morbilidades, la severidad de la enfermedad crítica, el estado funcional previo del paciente $y$, por último, habría que tener en cuenta sus preferencias respecto al tratamiento de soporte vital ${ }^{20}$.

Limitaciones del estudio son, por un lado, no disponer de datos sobre la calidad de vida y/o la dependencia de los pacientes, lo cual parece ser que influye directamente en el pronóstico a largo plazo de los pacientes mayores ${ }^{21}$. Otra limitación del estudio es no tener datos sobre la «limitación del esfuerzo terapéutico», la cual se decide más frecuentemente en pacientes mayores y con comorbilidades ${ }^{22}$.

En conclusión, a través de nuestro estudio podemos decir que en nuestro entorno la proporción de pacientes mayores que ingresan en la UCI aún es muy baja; que en la mortalidad de éstos no influye la duración de la estancia en la UCI (ya sea $<$ o $\geq 30$ días); y que la supervivencia al año de estos pacientes es alta. Todos estos resultados son suficientes, en nuestro entorno, para justificar la estancia prolongada de los pacientes mayores.

\section{Declaración de conflicto de intereses}

Los autores han declarado no tener ningún conflicto de intereses.

\section{BIBLIOGRAFÍA}

1. Stricker K, Rothen HU, Takala J. Resource use in the ICU: short- vs. long-term patients. Acta Anaesthesiol Scand. 2003;47: 508-15.

2. Bashour CA, Yared JP, Ryan TA, Rady MY, Mascha E, Leventhal MJ, et al. Long-term survival and functional capacity in cardiac surgery patients after prolonged intensive care. Crit Care Med. 2000;28:3847-53.

3. Heyland DK, Konopad E, Noseworthy TW, Johnston R, Gafni A. Is it 'worthwhile' to continue treating patients with a prolonged stay (> 14 days) in the ICU? An economic evaluation. Chest. 1998;114:192-8.

4. Hughes M, MacKirdy FN, Norrie J, Grant IS. Outcome of long-stay intensive care patients. Intensive Care Med. 2001;27:779-82.

5. Friedrich JO, Wilson G, Chant C. Long-term outcomes and clinical predictors of hospital mortality in very long stay intensive care unit patients: a cohort study. Crit Care. 2006;10:R59.

6. Montuclard L, Garrouste-Orgeas M, Timsit JF, Misset B, De Jonghe B, Carlet J. Outcome, functional autonomy, and quality of life of elderly patients with a long-term intensive care unit stay. Crit Care Med. 2000;28:3389-95.

7. Jakob SM, Rothen HU. Intensive care 1980-1995: change in patient characteristics, nursing workload and outcome. Intensive Care Med. 1997;23:1165-70.

8. De Rooij SE, Abu-Hanna A, Levi M, de Jonge E. Factors that predict outcome of intensive care treatment in very elderly patients: a review. Crit Care. 2005;9:R307-14

9. López-Messa JB. Envejecimiento y Medicina Intensiva. Med Intensiva. 2005;29:469-74.

10. García Lizana F, Manzano Alonso JL, Saavedra Santana P. Mortalidad y calidad de vida al año del alta de una unidad de cuidados intensivos en pacientes mayores de 65 años. Med Clin (Barc). 2001;116:521-5.

11. Martin CM, Hill AD, Burns K, Chen LM. Characteristics and outcomes for critically ill patients with prolonged intensive care unit stays. Crit Care Med. 2005;33:1922-7; quiz 1936.

12. Combes A, Costa MA, Trouillet JL, Baudot J, Mokhtari M, Gibert C, et al. Morbidity, mortality, and quality-of-life outcomes 
of patients requiring $>$ or $=14$ days of mechanical ventilation. Crit Care Med. 2003;31:1373-81.

13. Stricker KH, Cavegn R, Takala J, Rothen HU. Does ICU length of stay influence quality of life? Acta Anaesthesiol Scand. 2005;49:975-83.

14. Delle Karth G, Meyer B, Bauer S, Nikfardjam M, Heinz G. Outcome and functional capacity after prolonged intensive care unit stay. Wien Klin Wochenschr. 2006;118:390-6.

15. Arabi Y, Venkatesh S, Haddad S, Al Shimemeri A, Al Malik S. A prospective study of prolonged stay in the intensive care unit: predictors and impact on resource utilization. Int J Qual Health Care. 2002;14:403-10.

16. Weissman C. Analyzing the impact of long-term patients on ICU bed utilization. Intensive Care Med. 2000;26:1319-25.

17. Higgins TL, McGee WT, Steingrub JS, Rapoport J, Lemeshow S, Teres D. Early indicators of prolonged intensive care unit stay: impact of illness severity, physician staffing, and pre-intensive care unit length of stay. Crit Care Med. 2003;31:45-51.

18. Barie PS, Hydo LJ, Fischer E. Utility of illness severity scoring for prediction of prolonged surgical critical care. J Trauma. 1996;40:513-8; discussion 518-9.

19. Carrasco G, Pallarés A, Cabré L. Costes de la calidad en Medicina Intensiva. Guía para gestores clínicos. Med Intensiva. 2006;30:167-79.

20. Marik PE. Management of the critically ill geriatric patient. Crit Care Med. 2006;34 Suppl:S176-82.

21. Boumendil A, Somme D, Garrouste-Orgeas M, Guidet B. Should elderly patients be admitted to the intensive care unit? Intensive Care Med. 2007;33:1252-62.

22. Fernández R, Baigorri F, Artigas A. Limitación del esfuerzo terapéutico en Cuidados Intensivos. ¿Ha cambiado en el siglo XXI? Med Intensiva. 2005;29:338-41. 